\title{
Design, Synthesis, and Evaluation of Glycopeptides Containing Glucosamine
}

\author{
Guichao Xu' ${ }^{1}$, Xiaomin Wang ${ }^{2}$, Meng Zhang ${ }^{1}$, Li Zeng ${ }^{1}$, Hong Li ${ }^{1}$, Pengchao Gao ${ }^{1}$ and Jianwei Zhang ${ }^{1 *}$ \\ ${ }^{1}$ College of Pharmaceutical Sciences, Capital Medical University, Beijing 100069, PRC, China \\ ${ }^{2}$ Department of Physiology, Key Laboratory for Neurodegenerative Disorders of the Ministry of Education, Capital Medical University, Beijing 100069, PRC, China
}

\begin{abstract}
In this study, several novel peptidomimetics were designed and synthesized as potential anti-inflammatory agents. Their anti-inflammatory activity was evaluated using a xylene-induced ear edema model assay. Some of the compounds demonstrated inhibitory activity against xylene-induced inflammation similar to the positive control, aspirin.
\end{abstract}

Keywords: Amino sugar; Glycopeptide; Glucosamine; Inflammation

\section{Introduction}

Inflammation and various inflammatory disorders are widely prevalent all over the world. They afflict large percentage of the world's population. Inflammation is a normal protective response of mammalian tissues to a variety of hostile agents including infectious organisms, noxious chemicals, physical injury or tumor growth leading to local accumulation of plasmic fluid and blood cells. Although inflammation is a defense mechanism, the complex events and mediators involved in the inflammatory reaction can induce, maintain and aggravate many disorders. Inflammation are associated with many pathophysiology of various clinical conditions like arthritis, cancer and vascular diseases. Drugs used for the treatment of acute and chronic inflammatory disorders are usually directed at the inflammatory processes. Hence, the employment of anti-inflammatory agents may be helpful in the therapeutic treatment of those pathologies associated with inflammatory reactions. Through years of ingenious syntheses and structural modifications, which usually accompany design and development of new drug substances, many non-steroidal antiinflammatory agents have been prepared and marketed. These have been of immense help in the management of various inflammatory conditions like rheumatism, arthritis and breast pain. However, further development of a novel class of anti-inflammatory agents is needed.

The carbohydrates of glycoproteins have been subjected to an increasing interest during the last few years because they play important roles in numerous biological processes including modification of proteins, the immune response [1-3], cell adhesion [4-7], inflammation, tumor metastasis [8], and substrate- receptor recognition $[9,10]$. The effects of carbohydrates on biological activity and stability of glycoproteins has been studied previously $[11,12]$.

Carbohydrates that coat the surfaces of bacteria are capable of inducing an immune response that can recognize whole bacteria [13-16], and some functional glycoproteins that are expressed on tumour cell surfaces, have been shown to induce specific antitumour cell antibody responses in mice and patients [17]. There is a growing interest in carbohydrate mimetic peptides as vaccines to target cell surface polysaccharides of infectious bacteria and tumour [18].

Owing to the inherent complexity of carbohydrates, glycosylation can produce enormous structural diversity in proteins and induce a variety of functional changes. Since glycosyl amino acids and glycopeptides may be of value for medicinal chemistry, another important issue is their stability toward enzymatic degradation under physiological conditions. The field of glycobiology has grown explosively, and the interest in the development of methods for the synthesis of glycoconjugates is rapidly expanding as their biological and medical roles come into focus. Recent progress in this area has been remarkable, and relatively carbohydrate moieties have been covalently attached to amino acids for incorporation into glycopeptide sequences. Thus, many efforts have been devoted to establish easy and efficient methods for glycopeptide synthesis $[19,20]$.

The synthesis of monosaccharide conjugates is more straightforward than disaccharide or higher order oligosaccharide conjugates and this should facilitate a more rapid investigation of this possibility. We were interested to develop compounds reduced in carbohydrate character (monosaccharides). Amino sugars are widely distributed in living organisms and occur as constituents of glycoproteins, glycolipids, bacterial lipopolysaccharides, and proteoglycans. Glucosamine, an amino monosaccharide, is the most common amino sugar and is generally found as an $N$-acetylated and $\beta$-linked glycoside and is a natural component of glycoproteins found in connective tissues and gastrointestinal mucosal membranes. Glucosamine (GlcN) salts (sulfate and chloride) represent a new generation of drugs, which possess potentially chondroprotective or disease-modifying properties and were originally suggested to promote the repair of damaged cartilage. Since the first publication of Bohne in 1969 showing that GlcN can be used as a single pharmacologic agent to relieve the symptoms of osteoarthritis. Glucosamine has recently received a great deal of attention from the public as a potential treatment for OA [21]. Furthermore, the multiple antioxidant activity of glucosamine was evident as it showed pronounced reducing power, superoxide/ hydroxyl-radical scavenging ability. Because it is non-toxic, it may be a desired food supplement as a potential antioxidant. Experimental evidence was presented that glucosamine possess a unique range of anti-inflammatory activities and inhibit IL- $1 \beta$ - and TNF- $\alpha$-induced NO production in normal human articular chondrocytes. It is also a therapeutic agent for inflammatory bowel diseases [22].

Peptides related to Arg-Gly-Asp (RGD) are known to contribute various biologic functions. Integrin receptors constitute a large family of proteins with structural characteristics of noncovalent heterodimeric glycoproteins formed of $\alpha$ and $\beta$ subunits [23,24]. One important recognition site for many integrins is the arginine-glycine-asparagine

${ }^{*}$ Corresponding author: Jianwei Zhang, College of Pharmaceutical Sciences, Capital Medical University, Beijing 100069, PRC, China, Tel: 86-10-8391-1522; Fax: 86-10-8391-1533; E-mail: jwzhang2006@163.com

Received May 21, 2014; Accepted June 27, 2014; Published June 29, 2014

Citation: Xu G, Wang X, Zhang M, Zeng L, Li H, et al. (2014) Design, Synthesis, and Evaluation of Glycopeptides Containing Glucosamine. Med chem 4: 494-500. doi:10.4172/2161-0444.1000184

Copyright: ( 2014 Xu G, et al. This is an open-access article distributed under the terms of the Creative Commons Attribution License, which permits unrestricted use, distribution, and reproduction in any medium, provided the original author and source are credited. 
tripeptide sequence. Regarding RGD-dependent integrins, $\alpha \beta 3$ and $\alpha_{\mathrm{v}} \beta 5$ receptors have received increasing attention as therapeutic targets, as the integrins $\alpha_{\mathrm{v}} \beta 3$ and $\alpha_{\mathrm{v}} \beta 5$ can be expressed by both tumor cells and tumor endothelial cells [25-27]. It is speculated that drugs that inhibit the adhesive function of these integrins can inhibit tumor growth. Among selective receptor-targeting small peptides, integrinmediated RGD peptide appear attractive candidates. Moreover, it is well known that RGD peptide can suppress platelet aggregation by blocking the interaction between platelet and fibrinogen.

There is an increased interest in the use of hybrid molecules for drug discovery against a multitude of disease indications [28-32]. A twin drug bearing two different pharmacophoric entities is expected to bind to the respective receptors for each monomeric ligand and could elicit the corresponding effects derived from the individual receptors [33]. Furthermore, a twin drug can sometimes show an unexpected effect, which may not be predicted from each monomeric unit. The present work is towards the development and identification of new molecules as high potent anti- inflammatory agents.

Amide bond is very stable in buffer solution at pH 7.4 and in culture medium. Amides are an important functional group widely found in natural products, pharmaceuticals, and polymers. They may be prepared by coupling reactions between carboxylic acids and amines [34-36]. The synthetic design used for preparation of glycosyl amides was based upon a number of reasons:(1) glucosamine possess the biological activity; (2) $\mathrm{N}$-acetylglucosamine has several potential advantages over glucosamine as a potential therapeutic anti-inflammatory agent. In addition, it has been reported that some orally administered peptide analogues could be effectively absorbed through the intestinal peptide transport system. Amino acids are attractive because they possess structurally diverse side chains which allow to manipulate of the pharmacokinetics profiles of the compounds by using different amino acids. In this context, an interest has been increased in linking glucosamine with RGD peptide by the addition different lipophilic amino acid residues. Different lipophilic amino acids were used as building units for the preparation of a series tetrapeptide $\mathrm{RGDAA}$ ( $\mathrm{AA}=$ amino acid residues) as building blocks: these were RGD peptide derivatives $C$-linked via valine, phenyalanine and serine. In this study, the synthesis of peptidomimetics in which RGDAA were conjugated via an amide linkage to a monosaccharide (glucosamine) with variations in the peptide part were reported [32]. Glucosamine and $\mathrm{N}$-acetylglucosamine have been reported to possess interesting biological activity [33-37], therefore the creation of these compounds would be useful for further biological evaluation, as well as for detailed structure-activity studies and may have potential as drugs or as leads for drug development.

\section{Materials and Methods}

\section{General procedures}

Unless otherwise stated, all reactions were under a nitrogen atmosphere ( 1 bar). The agents used in this work were purchased from Sigma Chemical Co (USA). Optical rotations were determined with a Schmidt+Haensch Polartromic D instrument (Germany). IR spectra were recorded with an Avatar 330, Nicolet, USA spectrometer. ${ }^{1} \mathrm{H}$ and ${ }^{13} \mathrm{C}$ NMR spectra were recorded at $300 \mathrm{MHz}$ on a VXR-300 instrument or at $500 \mathrm{MHz}$ on a Bruker Am-500 instrument in DMSO-d6 with tetramethylsilane as internal standard and chemical shifts are expressed in ppm ( $\delta$ ). Chromatography was performed on Qingdao silica gel $\mathrm{H}$ (Qingdao of China). TLC analysis was carried out on silica gel $\mathrm{F}_{254}$. The purities ( $>95 \%$ ) of the intermediates and the products were confirmed by TLC (Merck silica gel plates of type $60 \mathrm{~F}_{254}, 0.25 \mathrm{~mm}$ layer thickness,
Germany) and HPLC (Waters, $\mathrm{C}_{8}$ column $4.6 \times 250 \mathrm{~mm}$, Ireland). MS was acquired on a Quattro Micro ZQ2000, Waters, USA instrument, $\mathrm{m} / \mathrm{z}$ values are reported. High-resolution mass spectra were recorded with microTOF-Q mass spectrometer.

\section{Syntheses}

$\mathrm{N}$-(Val-Asp(MeO)-Gly-Arg( $\left.\mathrm{NO}_{2}\right)$-Boc)-2-amino-1,3,4,6-tetraO-benzyl-2-deoxy-D-glucoamine: $\mathrm{HOBt}(800 \mathrm{mg}, 5.93 \mathrm{mmol})$ and DCC $(1.20 \mathrm{~g}, 5.83 \mathrm{mmol})$ were added to a solution of Boc- $\operatorname{Arg}\left(\mathrm{NO}_{2}\right)$ Gly-Asp(OMe)-Val-OH (3.48g, $5.76 \mathrm{mmol})$ in anhydrous THF (30 $\mathrm{mL}$ ) at $0^{\circ} \mathrm{C}$. The reaction mixture was stirred at $0^{\circ} \mathrm{C}$ for $30 \mathrm{~min}$. The solution of 2-amino-1,3,4,6-tetra-O-benzyl-2-deoxy-D-glucoamine $(2.95 \mathrm{~g}, 5.47 \mathrm{mmol})$ in anhydrous THF $(20 \mathrm{~mL})$ was added and adjusted to $\mathrm{pH} 9$ with $\mathrm{N}$-methylmorpholine. The reaction mixture obtained was keep at $0^{\circ} \mathrm{C}$ for $2 \mathrm{~h}$ followed by at room temperature for $24 \mathrm{~h}$. DCU formed was removed by filtration. The filtrate was evaporated under reduced pressure and the residue was dissolved in EtOAc $(50 \mathrm{~mL})$. The solution was washed successively with saturated $\mathrm{NaHCO}_{3}, 5 \% \mathrm{KHSO}_{4}$ and saturated $\mathrm{NaCl}$ and the organic phase was separated and dried over $\mathrm{Na}_{2} \mathrm{SO}_{4}$. Following filtration and evaporation under reduced pressure, the residue was purified by column chromatography $\left(20: 1 \mathrm{CH}_{2} \mathrm{Cl}_{2}\right.$ $\mathrm{MeOH})$ to provide Compound 9a $\left(4.23 \mathrm{~g}, 3.76 \mathrm{mmol}, 69 \%, \alpha:{ }^{2}=\right.$ 1.8:1). ${ }^{1} \mathrm{HNMR}\left(500 \mathrm{MHz}, \mathrm{DMSO}-d_{6}\right): \delta 8.48(1 \mathrm{H}, \mathrm{brs}, \mathrm{N}-\mathrm{H}), 8.35(1 \mathrm{H}$, $\mathrm{d}, \mathrm{J}=8.5 \mathrm{~Hz}, \mathrm{~N}-\mathrm{H}), 8.29(1 \mathrm{H}, \mathrm{d}, \mathrm{J}=9.0 \mathrm{~Hz}, \mathrm{~N}-\mathrm{H}), 8.24(1 \mathrm{H}, \mathrm{d}, \mathrm{J}=8.0$ $\mathrm{Hz}, \mathrm{N}-\mathrm{H}), 8.19(1 \mathrm{H}, \mathrm{d}, \mathrm{J}=9.0 \mathrm{~Hz}, \mathrm{~N}-\mathrm{H}), 8.15(1 \mathrm{H}, \mathrm{m}, \mathrm{N}-\mathrm{H}), 7.77(1 \mathrm{H}$, d, J = 9.0 Hz, N-H), $7.66(1 \mathrm{H}, \mathrm{d}, \mathrm{J}=9.0 \mathrm{~Hz}, \mathrm{~N}-\mathrm{H}), 7.44-7.15(19 \mathrm{H}, \mathrm{m}$, $\operatorname{Ar}-\mathrm{H}), 6.94(1 \mathrm{H}, \mathrm{t}, \mathrm{J}=6.9 \mathrm{~Hz}, \mathrm{Ar}-\mathrm{H}), 4.78(1 \mathrm{H}, \mathrm{m}, \mathrm{CH}), 4.77(1 \mathrm{H}, \mathrm{d}, \mathrm{J}=$ $4.0 \mathrm{~Hz}, \mathrm{H}-1 \alpha), 4.76(1 \mathrm{H}, \mathrm{d}, \mathrm{J}=8.5 \mathrm{~Hz}, \mathrm{H}-1 \beta), 4.70\left(4 \mathrm{H}, \mathrm{m}, \mathrm{CH}_{2} \mathrm{Ph}\right), 4.66$ $\left(4 \mathrm{H}, \mathrm{m}, \mathrm{CH}_{2} \mathrm{Ph}\right), 4.37\left(1 \mathrm{H}, \mathrm{d}, \mathrm{J}_{1}=9.0 \mathrm{~Hz}, \mathrm{~J}_{2}=6.0 \mathrm{~Hz}, \mathrm{CH}\right), 4.31(1 \mathrm{H}, \mathrm{dd}$, $\left.\mathrm{J}_{1}=9.0 \mathrm{~Hz}, \mathrm{~J}_{2}=6.0 \mathrm{~Hz}, \mathrm{CH}\right), 3.94\left(2 \mathrm{H}, \mathrm{dd}, \mathrm{J}_{1}=8.5 \mathrm{~Hz}, \mathrm{~J}_{2}=3.5 \mathrm{~Hz}, \mathrm{CH}_{2}\right)$ 3.83-3.64 (6H, m, CH H-2, H-3, H-4, H-5, H-6a), 3.59 ( $\left.3 \mathrm{H}, \mathrm{s}, \mathrm{CH}_{3}\right)$, $3.57(1 \mathrm{H}, \mathrm{m}, \mathrm{H}-6 \mathrm{~b}), 2.73(1 \mathrm{H}, \mathrm{m}, \mathrm{CH}), 2.54(1 \mathrm{H}, \mathrm{m}, \mathrm{CH}), 2.08(1 \mathrm{H}, \mathrm{m}$, $\mathrm{CH}), 1.65\left(1 \mathrm{H}, \mathrm{m}, \mathrm{CH}_{2}\right), 1.51\left(3 \mathrm{H}, \mathrm{m}, \mathrm{CH}_{2}\right), 1.38\left(9 \mathrm{H}, \mathrm{s}, \mathrm{CH}_{3}\right), 0.84(3 \mathrm{H}$, $\left.\mathrm{d}, \mathrm{J}=6.5 \mathrm{~Hz}, \mathrm{CH}_{3}\right), 0.77\left(3 \mathrm{H}, \mathrm{d}, \mathrm{J}=6.5 \mathrm{~Hz}, \mathrm{CH}_{3}\right), 0.76(3 \mathrm{H}, \mathrm{d}, \mathrm{J}=6.5 \mathrm{~Hz}$, $\left.\mathrm{CH}_{3}\right), 0.70\left(3 \mathrm{H}, \mathrm{d}, \mathrm{J}=6.5 \mathrm{~Hz}, \mathrm{CH}_{3}\right) ;{ }^{13} \mathrm{CNMR}\left(125 \mathrm{MHz}, \mathrm{CDCl}_{3}\right) \delta 172.7$, 171.4, 171.2, 171.0, 170.9, 170.3, 170.2, 169.4, 169.1, 159.8, 155.9, 139.1, 139.0, 138.7, 138.6, 138.5, 137.9, 137.7, 128.8, 128.7, 128.6, 128.5, 128.4, $128.3,128.2,128.1,127.9,96.7,79.9,79.4,79.9,78.7,74.7,74.6,74.3$, $72.8,70.9,69.1,69.0,58.1,57.8,54.4,53.5,52.0,51.9,49.8,42.6,42.4$, 37.1, 36.2, 31.4, 31.2, 29.6, 28.7, 25.2, 19.8, 19.6, 18.3, 17.9; IR $\left(\mathrm{cm}^{-1}\right.$, $\mathrm{KBr}$, neat): $3286,1643,741$; ESIMS $m / z$ 1148(M+Na); HRMS calcd for : $\left(\mathrm{C}_{57} \mathrm{H}_{76} \mathrm{~N}_{9} \mathrm{O}_{15}+1\right), m / z(1126.5455)$; found, $m / z(1126.5366)$.

$\mathrm{N}$-(Phe-Asp(OMe)-Gly-Arg( $\left.\mathrm{NO}_{2}\right)$-Boc)-2-amino-1,3,4,6-tetraO-benzyl-2-deoxy- $\boldsymbol{\alpha}$-D-glucosam-ine: $\mathrm{HOBt}(310 \mathrm{mg}, 2.29 \mathrm{mmol})$ and DCC (500 mg, $2.43 \mathrm{mmol})$ were added to a solution of Boc- $\operatorname{Arg}\left(\mathrm{NO}_{2}\right)$ Gly-Asp(OMe)-Phe-OH (1.46 g, $2.24 \mathrm{mmol}$ ) in anhydrous THF (10 $\mathrm{mL}$ ) at $0^{\circ} \mathrm{C}$. The reaction mixture was stirred at $0^{\circ} \mathrm{C}$ for $30 \mathrm{~min}$. The solution of 2-amino-1,3,4,6-tetra-O-benzyl-2-deoxy-D-glucoamine $(1.22 \mathrm{~g}, 2.26 \mathrm{mmol}))$ in anhydrous THF $(20 \mathrm{~mL})$ was added and adjusted to $\mathrm{pH} 9$ with $\mathrm{N}$-methylmorpholine. The reaction mixture obtained was keep at $0^{\circ} \mathrm{C}$ for $2 \mathrm{~h}$ followed by at room temperature for $24 \mathrm{~h}$. DCU formed was removed by filtration. The filtrate was evaporated under reduced pressure and the residue was dissolved in EtOAc $(50 \mathrm{~mL})$. The solution was washed successively with saturated $\mathrm{NaHCO}_{3}, 5 \% \mathrm{KHSO}_{4}$ and saturated $\mathrm{NaCl}$ and the organic phase was separated and dried over $\mathrm{Na}_{2} \mathrm{SO}_{4}$. Following filtration and evaporation under reduced pressure, the residue was purified by column chromatography $\left(20: 1 \mathrm{CH}_{2} \mathrm{Cl}_{2}-\right.$ $\mathrm{MeOH})$ to provide Compound $9 \mathrm{~b}(1.8 \mathrm{~g}, 1.53 \mathrm{mmol}, 79 \%)$. [a] ${ }^{25}{ }_{\mathrm{D}}=$ $+21.5(\mathrm{C}=0.7, \mathrm{MeOH}) ; \mathrm{IR}\left(\mathrm{cm}^{-1}, \mathrm{KBr}\right.$, neat $): 3287,1636 ;{ }^{1} \mathrm{HNMR}(500$ $\left.\mathrm{MHz}, \mathrm{DMSO}-d_{6}\right): \delta 8.33(1 \mathrm{H}, \mathrm{d}, \mathrm{J}=8.4 \mathrm{~Hz}, \mathrm{~N}-\mathrm{H}), 8.03(1 \mathrm{H}, \mathrm{d}, \mathrm{J}=8.4$ $\mathrm{Hz}, \mathrm{N}-\mathrm{H}), 7.94(1 \mathrm{H}, \mathrm{d}, \mathrm{J}=8.4 \mathrm{~Hz}, \mathrm{~N}-\mathrm{H}), 7.41-7.14(25 \mathrm{H}, \mathrm{m}, \mathrm{Ar}-\mathrm{H}), 4.79$ $(1 \mathrm{H}, \mathrm{d}, \mathrm{J}=3.0 \mathrm{~Hz}, \mathrm{H}-1), 4.78-4.69\left(7 \mathrm{H}, \mathrm{m}, \mathrm{CH}_{2} \mathrm{Ph}, \mathrm{CH}\right), 4.63(1 \mathrm{H}, \mathrm{m}$, 
$\mathrm{CH}), 4.56\left(2 \mathrm{H}, \mathrm{m}, \mathrm{CH}_{2} \mathrm{Ph}\right), 3.99(2 \mathrm{H}, \mathrm{m}, \mathrm{CH}, \mathrm{H}-2), 3.85(1 \mathrm{H}, \mathrm{t}, \mathrm{J}=9.5$ $\mathrm{Hz}, \mathrm{H}-3), 3.79(1 \mathrm{H}, \mathrm{m}, \mathrm{H}-5), 3.68\left(4 \mathrm{H}, \mathrm{m}, \mathrm{CH}_{2}, \mathrm{H}-6 \mathrm{a}, \mathrm{H}-6 \mathrm{~b}\right), 3.58(1 \mathrm{H}$, $\mathrm{t}, \mathrm{J}=9.5 \mathrm{~Hz}, \mathrm{H}-4), 3.52\left(3 \mathrm{H}, \mathrm{s}, \mathrm{CH}_{3}\right), 3.13\left(2 \mathrm{H}, \mathrm{m}, \mathrm{CH}_{2}\right), 2.94(1 \mathrm{H}, \mathrm{dd}$, $\left.\mathrm{J}_{1}=13.2 \mathrm{~Hz}, \mathrm{~J}_{2}=3.3 \mathrm{~Hz}, \mathrm{CH}_{2}\right), 2.76\left(1 \mathrm{H}, \mathrm{dd}, \mathrm{J}_{1}=13.2 \mathrm{~Hz}, \mathrm{~J}_{2}=3.0 \mathrm{~Hz}\right.$, $\left.\mathrm{CH}_{2}\right), 2.64\left(1 \mathrm{H}, \mathrm{dd}, \mathrm{J}_{1}=16.2 \mathrm{~Hz}, \mathrm{~J}_{2}=6.0 \mathrm{~Hz}, \mathrm{CH}_{2}\right), 2.44\left(1 \mathrm{H}, \mathrm{dd}, \mathrm{J}_{1}=16.2\right.$ $\left.\mathrm{Hz}, \mathrm{J}_{2}=7.8 \mathrm{~Hz}, \mathrm{CH}_{2}\right), 1.66\left(1 \mathrm{H}, \mathrm{m}, \mathrm{CH}_{2}\right), 1.52\left(3 \mathrm{H}, \mathrm{m}, \mathrm{CH}_{2}\right), 1.38(9 \mathrm{H}$, s, $\left.\mathrm{CH}_{3}\right) ;{ }^{13} \mathrm{CNMR}\left(125 \mathrm{MHz}, \mathrm{CDCl}_{3}\right) \delta 172.8,171.4,171.0,170.2,169.1$, 159.6, 155.9, 139.1, 139.0, 138.7, 138.6, 138.0, 137.9, 129.6, 128.8, 128.7, $128.5,128.4,128.3,128.2,128.1,127.9,127.8,127.7,126.7,96.7,79.8$, 78.9, 78.8, 74.6, 74.4, 72.8, 71.0, 69.1, 54.4, 54.3, 53.5, 51.9, 49.5, 42.3, 38.0, 36.2, 29.5, 28.6, 25.2; ESIMS $m / z$ 1196(M+Na); HRMS calcd for: $\left(\mathrm{C}_{61} \mathrm{H}_{76} \mathrm{~N}_{9} \mathrm{O}_{15}+1\right), m / z(1174.5455)$; found, $m / z(1174.5705)$.

$\mathrm{N}$-(Ser(OBn)-Asp(OMe)-Gly-Arg( $\left.\mathrm{NO}_{2}\right)$-Boc)-2-amino-1,3,4,6tetra-O-benzyl-2-deoxy- $\boldsymbol{\alpha}$-D-glu-cosamine: HOBt (135 mg, 1.00 $\mathrm{mmol})$ and DCC $(200 \mathrm{mg}, 0.97 \mathrm{mmol})$ were added to a solution of Boc-Arg( $\left.\mathrm{NO}_{2}\right)$-Gly-Asp(OMe)-Ser(OBn)-OH (560 mg, $0.82 \mathrm{mmol}$ ) in anhydrous THF $(15 \mathrm{~mL})$ at $0^{\circ} \mathrm{C}$. The reaction mixture was stirred at $0^{\circ} \mathrm{C}$ for $30 \mathrm{~min}$. The solution of 2-amino-1,3,4,6-tetra-O-benzyl-2-deoxy-Dglucoamine (493 mg, $0.91 \mathrm{mmol}$ ) in anhydrous THF $(5 \mathrm{~mL})$ was added and adjusted to $\mathrm{pH} 9$ with $\mathrm{N}$-methylmorpholine. The reaction mixture obtained was keep at $0^{\circ} \mathrm{C}$ for $2 \mathrm{~h}$ followed by at room temperature for 24 h. DCU formed was removed by filtration. The filtrate was evaporated under reduced pressure and the residue was dissolved in EtOAc (50 $\mathrm{mL}$ ). The solution was washed successively with saturated $\mathrm{NaHCO}_{3}$, $5 \% \mathrm{KHSO}_{4}$ and saturated $\mathrm{NaCl}$ and the organic phase was separated and dried over $\mathrm{Na}_{2} \mathrm{SO}_{4}$. Following filtration and evaporation under reduced pressure, the residue was purified by column chromatography $\left(20: 1 \mathrm{CH}_{2} \mathrm{Cl}_{2}-\mathrm{MeOH}\right)$ to provide Compound 9c (600 mg, $0.50 \mathrm{mmol}$, 61\%). $[\alpha]_{\mathrm{D}}^{25}=+28.2$ (C=1.0, $\left.\mathrm{MeOH}\right) ; \mathrm{IR}\left(\mathrm{cm}^{-1}, \mathrm{KBr}\right.$, neat): 3287,1651 ; ${ }^{1} \mathrm{HNMR}\left(500 \mathrm{MHz}, \mathrm{DMSO}-d_{6}\right): \delta 8.23(1 \mathrm{H}, \mathrm{d}, \mathrm{J}=8.7 \mathrm{~Hz}, \mathrm{~N}-\mathrm{H}), 8.10$ $(1 \mathrm{H}, \mathrm{d}, \mathrm{J}=8.1 \mathrm{~Hz}, \mathrm{~N}-\mathrm{H}), 7.39-7.14(25 \mathrm{H}, \mathrm{m}, \mathrm{Ar}-\mathrm{H}), 4.77(1 \mathrm{H}, \mathrm{d}, \mathrm{J}=3.5$ $\mathrm{Hz}, \mathrm{H}-1), 4.76(1 \mathrm{H}, \mathrm{m}, \mathrm{CH}), 4.72\left(5 \mathrm{H}, \mathrm{m}, \mathrm{CH}_{2} \mathrm{Ph}, \mathrm{CH}\right), 4.52(4 \mathrm{H}, \mathrm{m}$, $\left.\mathrm{CH}_{2} \mathrm{Ph}\right), 4.37\left(2 \mathrm{H}, \mathrm{m}, \mathrm{CH}_{2} \mathrm{Ph}\right), 3.96(2 \mathrm{H}, \mathrm{m}, \mathrm{CH}, \mathrm{H}-2), 3.82(1 \mathrm{H}, \mathrm{t}, \mathrm{J}=$ $8.7 \mathrm{~Hz}, \mathrm{H}-3), 3.78$ (1H, m, H-5), $3.72(2 \mathrm{H}, \mathrm{m}, \mathrm{H}-6 \mathrm{a}, \mathrm{H}-6 \mathrm{~b}), 3.66(2 \mathrm{H}$, $\left.\mathrm{m}, \mathrm{CH}_{2}\right), 3.59\left(3 \mathrm{H}, \mathrm{m}, \mathrm{H}-4, \mathrm{CH}_{2}\right), 3.54\left(3 \mathrm{H}, \mathrm{s}, \mathrm{CH}_{3}\right), 3.12\left(2 \mathrm{H}, \mathrm{m}, \mathrm{CH}_{2}\right)$, $2.74\left(1 \mathrm{H}, \mathrm{dd}, \mathrm{J}_{1}=16.2 \mathrm{~Hz}, \mathrm{~J}_{2}=6.0 \mathrm{~Hz}, \mathrm{CH}_{2}\right), 2.57\left(1 \mathrm{H}, \mathrm{dd}, \mathrm{J}_{1}=16.2 \mathrm{~Hz}, \mathrm{~J}_{2}\right.$ $\left.=6.0 \mathrm{~Hz}, \mathrm{CH}_{2}\right), 1.65\left(1 \mathrm{H}, \mathrm{m}, \mathrm{CH}_{2}\right), 1.51\left(3 \mathrm{H}, \mathrm{m}, \mathrm{CH}_{2}\right), 1.38\left(9 \mathrm{H}, \mathrm{s}, \mathrm{CH}_{3}\right)$; ${ }^{13} \mathrm{CNMR}\left(125 \mathrm{MHz}, \mathrm{CDCl}_{3}\right) \delta 172.7,171.0,170.5,169.7,169.2,159.6$, $155.9,139.1,138.7,138.6,138.5,137.9,128.7,128.6,128.5,128.3,128.2$, $128.1,127.9,127.8,127.7,96.7,79.8,78.8,78.6,74.6,74.3,72.8,72.5$, 71.0, 70.3, 69.1, 66.5, 55.5, 54.3, 53.5, 53.4, 51.9, 49.6, 42.4, 36.5, 29.5, 28.6, 25.1; ESIMS $m / z 1204(\mathrm{M}+1)$; HRMS calcd for: $\left(\mathrm{C}_{62} \mathrm{H}_{78} \mathrm{~N}_{9} \mathrm{O}_{16}+1\right)$, $\mathrm{m} / z$ (1204.5561); found, $\mathrm{m} / z$ (1204.5747).

$\mathrm{N}$-(Val-Asp-Gly-Arg( $\left.\mathrm{NO}_{2}\right)$-Boc)-2-amino-1,3,4,6-tetra-Obenzyl-2-deoxy-a-D-glucosamine: The solution of 9a (1.50 g, 1.33 $\mathrm{mmol})$ in $2 \mathrm{M} \mathrm{NaOH}(4 \mathrm{~mL})$ in methanol $(20 \mathrm{~mL})$ and dioxane $(60$ $\mathrm{mL}$ ) was stirred at $0^{\circ} \mathrm{C}$ until all the starting material was consumed as indicated by TLC $(24 \mathrm{hr})$. The reaction mixture was neutralized ( $\mathrm{pH}$ 7) with $\mathrm{KHSO}_{4}$ and evaporated to remove the solvent. The residue was added water at $0^{\circ} \mathrm{C}(50 \mathrm{~mL})$ and $\mathrm{KHSO}_{4}$ to $\mathrm{pH} 2$. Following filtration, the residue was purified by column chromatography $\left(15: 1 \mathrm{CH}_{2} \mathrm{Cl}_{2}-\right.$ $\mathrm{MeOH}$ ) to provide Compound 10a $\mathrm{a}$-anomer (300 $\mathrm{mg}, 0.270 \mathrm{mmol}$, $74 \%)$ and 10a $\beta$-anomer (1.1g, $0.990 \mathrm{mmol}, 20 \%)$. 10a $\alpha$-anomer : [a] ${ }^{25}{ }_{\mathrm{D}}=+88.6(\mathrm{C}=0.2, \mathrm{MeOH}) ;{ }^{1} \mathrm{HNMR}\left(500 \mathrm{MHz}, \mathrm{DMSO}-d_{6}\right): \delta 7.40-$ $7.15(20 \mathrm{H}, \mathrm{m}, \mathrm{Ar}-\mathrm{H}), 4.77(1 \mathrm{H}, \mathrm{m}, \mathrm{CH}), 4.76(1 \mathrm{H}, \mathrm{d}, \mathrm{J}=3.5 \mathrm{~Hz}, \mathrm{H}-1)$, $4.68\left(4 \mathrm{H}, \mathrm{m}, \mathrm{CH}_{2} \mathrm{Ph}\right), 4.53\left(4 \mathrm{H}, \mathrm{m}, \mathrm{CH}_{2} \mathrm{Ph}\right), 4.32(1 \mathrm{H}, \mathrm{m}, \mathrm{CH}), 3.95$ $\left(2 \mathrm{H}, \mathrm{m}, \mathrm{CH}_{2}\right), 3.83(1 \mathrm{H}, \mathrm{m}, \mathrm{CH}), 3.80(2 \mathrm{H}, \mathrm{m}, \mathrm{H}-2, \mathrm{H}-3), 3.67(3 \mathrm{H}$, m, H-4, H-6a, H-6b), $3.56(1 \mathrm{H}, \mathrm{q}, \mathrm{J}=9.0 \mathrm{~Hz}, \mathrm{H}-5), 3.13\left(2 \mathrm{H}, \mathrm{m}, \mathrm{CH}_{2}\right)$, $2.67\left(1 \mathrm{H}, \mathrm{m}, \mathrm{CH}_{2}\right), 2.56\left(1 \mathrm{H}, \mathrm{m}, \mathrm{CH}_{2}\right), 1.98(1 \mathrm{H}, \mathrm{m}, \mathrm{CH}), 1.66(1 \mathrm{H}$, $\left.\mathrm{m}, \mathrm{CH}_{2}\right), 1.51\left(3 \mathrm{H}, \mathrm{m}, \mathrm{CH}_{2}\right), 1.38\left(9 \mathrm{H}, \mathrm{s}, \mathrm{CH}_{3}\right), 0.80\left(6 \mathrm{H}, \mathrm{m}, \mathrm{CH}_{3}\right)$; ${ }^{13} \mathrm{CNMR}\left(125 \mathrm{MHz}, \mathrm{CDCl}_{3}\right) \delta 172.7,171.4,170.6,169.3,168.9,159.6$,
$155.9,139.1,139.0,138.7,138.6,137.9,128.8,128.7,128.5,128.4,128.3$, $128.2,128.1,127.9,127.8,96.7,79.8,79.4,78.9,78.7,74.6,74.4,72.8$, $70.9,69.1,57.9,54.3,53.5,49.9,42.3,37.2,36.2,31.1,29.6,28.7,25.0$, 19.8, 19.7, 18.3, 17.8; IR ( $\mathrm{cm}^{-1}, \mathrm{KBr}$, neat): 3287, 1643, 741; ESIMS $\mathrm{m} / z$ 1111(M); HRMS calcd for : $\left(\mathrm{C}_{56} \mathrm{H}_{72} \mathrm{~N}_{9} \mathrm{O}_{15}\right), \mathrm{m} / z$ (1110.515336); found, $\mathrm{m} / z$ (1110.5226). 10a $\beta$-anomer: $[\alpha]{ }_{\mathrm{D}}^{25}=+51.4(\mathrm{C}=0.1, \mathrm{MeOH})$; IR ( $\mathrm{cm}^{-1}, \mathrm{KBr}$, neat): $3287,1643,741 ;{ }^{1} \mathrm{HNMR}$ (500 MHz, DMSO- $\left.d_{6}\right): \delta$ 7.39-7.16 (20H, m, Ar-H), $4.79(1 \mathrm{H}, \mathrm{d}, \mathrm{J}=11.4 \mathrm{~Hz}, \mathrm{H}-1), 4.76(1 \mathrm{H}, \mathrm{m}$, $\mathrm{CH}), 4.69\left(3 \mathrm{H}, \mathrm{m}, \mathrm{CH}_{2} \mathrm{Ph}\right), 4.55\left(5 \mathrm{H}, \mathrm{m}, \mathrm{CH}_{2} \mathrm{Ph}\right), 4.50(1 \mathrm{H}, \mathrm{m}, \mathrm{CH}$ ), $4.32(1 \mathrm{H}, \mathrm{m}, \mathrm{CH}), 3.95\left(2 \mathrm{H}, \mathrm{m}, \mathrm{CH}_{2}\right), 3.77(3 \mathrm{H}, \mathrm{m}, \mathrm{CH}, \mathrm{H}-2, \mathrm{H}-3)$, 3.67 (3H, m, H-4, H-6a, H-6b), $3.55(1 \mathrm{H}, \mathrm{m}, \mathrm{H}-5), 3.14\left(2 \mathrm{H}, \mathrm{m}, \mathrm{CH}_{2}\right)$, $2.59\left(2 \mathrm{H}, \mathrm{m}, \mathrm{CH}_{2}\right), 1.92(1 \mathrm{H}, \mathrm{m}, \mathrm{CH}), 1.68\left(1 \mathrm{H}, \mathrm{m}, \mathrm{CH}_{2}\right), 1.51(3 \mathrm{H}, \mathrm{m}$, $\left.\mathrm{CH}_{2}\right), 1.38\left(9 \mathrm{H}, \mathrm{s}, \mathrm{CH}_{3}\right), 0.77\left(3 \mathrm{H}, \mathrm{d}, \mathrm{J}=6.5 \mathrm{~Hz}, \mathrm{CH}_{3}\right), 0.71(3 \mathrm{H}, \mathrm{d}, \mathrm{J}=$ $\left.6.5 \mathrm{~Hz}, \mathrm{CH}_{3}\right) ;{ }^{13} \mathrm{CNMR}\left(125 \mathrm{MHz}, \mathrm{CDCl}_{3}\right) \delta 173.9,172.5,171.9,170.6$, 169.7, 168.5, 159.6, 155.8, 139.2, 138.7, 138.6, 137.9, 137.8, 128.8, 128.7, $128.6,128.5,128.4,128.3,128.2,128.1,128.0,127.9,127.8,127.7,96.6$, 79.3, 79.0, 78.6, 74.6, 74.3, 72.8, 70.9, 69.1, 68.9, 57.9, 54.3, 53.5, 42.3, $40.8,38.6,31.3,29.7,28.7,25.4,19.8,19.6,18.5,18.0,17.9$; ESIMS $m / z$ 1111(M); HRMS calcd for: $\left(\mathrm{C}_{56} \mathrm{H}_{72} \mathrm{~N}_{9} \mathrm{O}_{15}-1\right), \mathrm{m} / z$ (1110.5153); found, $\mathrm{m} / z(1110.5176)$.

$\mathrm{N}$-(Phe-Asp-Gly-Arg( $\left.\mathrm{NO}_{2}\right)$-Boc)-2-amino-1,3,4,6-tetra$\boldsymbol{O}$-benzyl-2-deoxy-a-D-glucosamine: The solution of $9 \mathrm{~b}(1.38 \mathrm{~g}$, $1.17 \mathrm{mmol})$ in $2 \mathrm{M} \mathrm{NaOH}(2 \mathrm{~mL})$ in methanol $(10 \mathrm{~mL})$ and dioxane $(30$ $\mathrm{mL}$ ) was stirred at $0^{\circ} \mathrm{C}$ until all the starting material was consumed as indicated by TLC $(24 \mathrm{hr})$. The reaction mixture was neutralized ( $\mathrm{pH}$ 7) with $\mathrm{KHSO}_{4}$ and evaporated to remove the solvent. The residue was added water at $0^{\circ} \mathrm{C}(50 \mathrm{~mL})$ and $\mathrm{KHSO}_{4}$ to $\mathrm{pH} 2$. Following filtration, the residue was purified by column chromatography $\left(15: 1 \mathrm{CH}_{2} \mathrm{Cl}_{2}-\mathrm{MeOH}\right)$ to provide Compound $10 \mathrm{~b}(0.950 \mathrm{~g}, 0.820 \mathrm{mmol}, 70 \%)$. [a] ${ }_{\mathrm{D}}^{25}=+49.2$ $(\mathrm{C}=0.2, \mathrm{MeOH}) ; \mathrm{IR}\left(\mathrm{cm}^{-1}, \mathrm{KBr}\right.$, neat): 3294, 1643, 741; ${ }^{1} \mathrm{HNMR}(300$ $\left.\mathrm{MHz}, \mathrm{DMSO}-d_{6}\right): \delta 8.49(1 \mathrm{H}, \mathrm{brs}, \mathrm{N}-\mathrm{H}), 8.22(1 \mathrm{H}, \mathrm{d}, \mathrm{J}=6.1 \mathrm{~Hz}, \mathrm{~N}-\mathrm{H})$, $7.99(1 \mathrm{H}$, brs, N-H), 7.40-7.18 $(25 \mathrm{H}, \mathrm{m}, \mathrm{Ar}-\mathrm{H}), 6.93(1 \mathrm{H}, \mathrm{d}, \mathrm{J}=7.5 \mathrm{~Hz}$, $\mathrm{N}-\mathrm{H}), 4.78(2 \mathrm{H}, \mathrm{m}, \mathrm{CH}), 4.72(1 \mathrm{H}, \mathrm{d}, \mathrm{J}=3.3 \mathrm{~Hz}, \mathrm{H}-1), 4.70(2 \mathrm{H}, \mathrm{m}$, $\left.\mathrm{CH}_{2} \mathrm{Ph}\right), 4.54\left(6 \mathrm{H}, \mathrm{m}, \mathrm{CH}_{2} \mathrm{Ph}\right), 3.88(4 \mathrm{H}, \mathrm{m}, \mathrm{CH}, \mathrm{H}-2, \mathrm{H}-3, \mathrm{H}-5), 3.62$ $\left(5 \mathrm{H}, \mathrm{m}, \mathrm{CH}_{2}, \mathrm{H}-4, \mathrm{H}-6 \mathrm{a}, \mathrm{H}-6 \mathrm{~b}\right), 3.13\left(2 \mathrm{H}, \mathrm{m}, \mathrm{CH}_{2}\right), 2.94(1 \mathrm{H}, \mathrm{m}, \mathrm{CH})$, $2.68(2 \mathrm{H}, \mathrm{t}, \mathrm{J}=12.0 \mathrm{~Hz}, \mathrm{CH}), 2.39(1 \mathrm{H}, \mathrm{m}, \mathrm{CH}), 1.70\left(1 \mathrm{H}, \mathrm{m}, \mathrm{CH}_{2}\right), 1.51$ $\left(3 \mathrm{H}, \mathrm{m}, \mathrm{CH}_{2}\right), 1.37\left(9 \mathrm{H}, \mathrm{s}, \mathrm{CH}_{3}\right) ;{ }^{13} \mathrm{CNMR}\left(125 \mathrm{MHz}, \mathrm{CDCl}_{3}\right) \delta 172.5$, 172.1, 172.0, 169.7, 168.6, 159.6, 155.8, 139.2, 139.1, 138.7, 138.6, 138.5, $138.2,137.9,129.5,129.4,128.8,128.7,128.6,128.5,128.4,128.3,128.2$, 128.1, 128.0, 127.9, 127.8, 127.7, 126.6, 96.8, 79.7, 78.9, 78.7, 74.6, 74.4, $72.8,71.0,69.1,54.4,53.6,53.5,49.6,42.1,38.1,29.8,28.7,25.2$; ESIMS $\mathrm{m} / z$ 1158(M-1); HRMS calcd for: $\left(\mathrm{C}_{60} \mathrm{H}_{72} \mathrm{~N}_{9} \mathrm{O}_{15}-1\right), \mathrm{m} / z(1158.5153)$; found, $m / z$ (1158.5303).

$\mathrm{N}$-(Ser-Asp-Gly-Arg( $\left.\mathrm{NO}_{2}\right)$-Boc)-2-amino-1,3,4,6-tetra-Obenzyl-2-deoxy- $\boldsymbol{\alpha}$-D-glucosamine: The solution of $9 \mathrm{c}$ (200 mg,0.166 $\mathrm{mmol})$ in $2 \mathrm{M} \mathrm{NaOH}(0.8 \mathrm{~mL})$ in methanol $(50 \mathrm{~mL})$ and dioxane $(15$ $\mathrm{mL}$ ) was stirred at $0^{\circ} \mathrm{C}$ until all the starting material was consumed as indicated by TLC $(24 \mathrm{hr})$. The reaction mixture was neutralized ( $\mathrm{pH}$ 7) with $\mathrm{KHSO}_{4}$ and evaporated to remove the solvent. The residue was added water at $0^{\circ} \mathrm{C}(50 \mathrm{~mL})$ and $\mathrm{KHSO}_{4}$ to $\mathrm{pH}$ 2. Following filtration, the residue was purified by column chromatography $\left(15: 1 \mathrm{CH}_{2} \mathrm{Cl}_{2}\right.$ $\mathrm{MeOH})$ to provide Compound 10c (147 mg, $0.123 \mathrm{mmol}, 75 \%)$. [a] ${ }_{\mathrm{D}}^{25}=+24.4(\mathrm{C}=0.2, \mathrm{MeOH}) ; \mathrm{IR}\left(\mathrm{cm}^{-1}, \mathrm{KBr}\right.$, neat $): 3302,1651 ;{ }^{1} \mathrm{HNMR}$ $\left(500 \mathrm{MHz}, \mathrm{DMSO}-d_{6}\right): \delta 8.46(1 \mathrm{H}, \mathrm{d}, \mathrm{J}=8.0 \mathrm{~Hz}, \mathrm{~N}-\mathrm{H}), 8.26(1 \mathrm{H}, \mathrm{d}, \mathrm{J}$ $=6.5 \mathrm{~Hz}, \mathrm{~N}-\mathrm{H}), 7.39-7.14(25 \mathrm{H}, \mathrm{m}, \mathrm{Ar}-\mathrm{H}), 4.78(2 \mathrm{H}, \mathrm{m}, \mathrm{H}-1, \mathrm{CH})$, $4.68\left(4 \mathrm{H}, \mathrm{m}, \mathrm{CH}_{2} \mathrm{Ph}\right), 4.50\left(5 \mathrm{H}, \mathrm{m}, \mathrm{CH}, \mathrm{CH}_{2} \mathrm{Ph}\right), 4.35\left(2 \mathrm{H}, \mathrm{m}, \mathrm{CH}_{2} \mathrm{Ph}\right)$, $3.98(2 \mathrm{H}, \mathrm{m}, \mathrm{CH}, \mathrm{H}-2), 3.80\left(3 \mathrm{H}, \mathrm{m}, \mathrm{H}-3, \mathrm{CH}_{2}\right), 3.64(3 \mathrm{H}, \mathrm{m}, \mathrm{H}-5$, H-6a, H-6b), $3.53\left(3 \mathrm{H}, \mathrm{m}, \mathrm{H}-4, \mathrm{CH}_{2}\right), 3.15\left(2 \mathrm{H}, \mathrm{m}, \mathrm{CH}_{2}\right), 3.02(1 \mathrm{H}, \mathrm{m}$, $\left.\mathrm{CH}_{2}\right), 2.65\left(1 \mathrm{H}, \mathrm{m}, \mathrm{CH}_{2}\right), 1.69\left(1 \mathrm{H}, \mathrm{m}, \mathrm{CH}_{2}\right), 1.52\left(3 \mathrm{H}, \mathrm{m}, \mathrm{CH}_{2}\right), 1.38$ $\left(9 \mathrm{H}, \mathrm{s}, \mathrm{CH}_{3}\right) ;{ }^{13} \mathrm{CNMR}\left(125 \mathrm{MHz}, \mathrm{CDCl}_{3}\right) \delta 172.6,170.2,168.5,159.6$, $155.8,139.2,138.7,138.6,138.5,137.9,137.8,128.8,128.5,128.4,128.3$, 
$128.2,128.1,127.9,79.7,78.7,78.6,74.6,74.4,72.8,72.5,70.9,70.5$, 69.1, 68.9, 54.3, 53.6, 51.9, 49.6, 42.2, 36.5, 29.6, 28.7, 25.2; ESIMS $m / z$ 1189(M); HRMS calcd for: $\left(\mathrm{C}_{61} \mathrm{H}_{74} \mathrm{~N}_{9} \mathrm{O}_{16}-1\right), m / z$ (1188.5259); found, $m / z(1188.5345)$.

$\boldsymbol{N}$-(Val-Asp-Gly-Arg)-2-amino-2-deoxy-a-D-glucosamine: The solution of 10a $(30 \mathrm{mg}, 0.0270 \mathrm{mmol})$ in TFA $(2.1 \mathrm{~mL})$ and $\mathrm{CF}_{3} \mathrm{SO}_{3} \mathrm{H}$ $(0.7 \mathrm{~mL})$ was stirred at $0^{\circ} \mathrm{C}$ until all the starting material was consumed as indicated by TLC $(3 \mathrm{hr})$. The reaction mixture was added $\mathrm{Et}_{2} \mathrm{O}(80 \mathrm{~mL})$, the precipitate was collected by filtration that was added $\mathrm{NH}_{3} \cdot \mathrm{H}_{2} \mathrm{O}$ to $\mathrm{pH}=9$. Subsequently, The residue was added $\mathrm{HAc}$ to $\mathrm{pH}=4$ and purified by column chromatography (Sephadex G-10) to provide Compound $11 \mathrm{a}(6.2 \mathrm{mg}, 0.008 \mathrm{mmol}, 38 \%)$. IR ( $\mathrm{cm}^{-1}, \mathrm{KBr}$, neat): 3132,1678 ; ${ }^{1} \mathrm{HNMR}\left(500 \mathrm{MHz}, \mathrm{DMSO}-d_{6}\right): \delta 4.21-3.81(7 \mathrm{H}, \mathrm{m}, \mathrm{CH}), 3.81-3.42$ $(5 \mathrm{H}, \mathrm{m}, \mathrm{CH}), 3.04\left(2 \mathrm{H}, \mathrm{m}, \mathrm{CH}_{2}\right), 2.21\left(2 \mathrm{H}, \mathrm{m}, \mathrm{CH}_{2}\right), 1.90(1 \mathrm{H}, \mathrm{m}, \mathrm{CH}$ ), $1.52\left(4 \mathrm{H}, \mathrm{m}, \mathrm{CH}_{2}\right), 0.84\left(6 \mathrm{H}, \mathrm{d}, \mathrm{J}=6.0 \mathrm{~Hz}, \mathrm{CH}_{3}\right), 3.53(3 \mathrm{H}, \mathrm{m}, \mathrm{H}-4$, $\left.\mathrm{CH}_{2}\right), 3.15\left(2 \mathrm{H}, \mathrm{m}, \mathrm{CH}_{2}\right), 3.02\left(1 \mathrm{H}, \mathrm{m}, \mathrm{CH}_{2}\right), 2.65\left(1 \mathrm{H}, \mathrm{m}, \mathrm{CH}_{2}\right), 1.69$ $\left(1 \mathrm{H}, \mathrm{m}, \mathrm{CH}_{2}\right), 1.52\left(3 \mathrm{H}, \mathrm{m}, \mathrm{CH}_{2}\right), 1.38\left(9 \mathrm{H}, \mathrm{s}, \mathrm{CH}_{3}\right) ;{ }^{13} \mathrm{CNMR}(125 \mathrm{MHz}$, $\left.\mathrm{CDCl}_{3}\right) \delta 172.6,170.2,168.5,159.6,155.8,139.2,138.7,138.6,138.5$, $137.9,137.8,128.8,128.5,128.4,128.3,128.2,128.1,127.9,79.7,78.7$, 78.6, 74.6, 74.4, 72.8, 72.5, 70.9, 70.5, 69.1, 68.9, 54.3, 53.6, 51.9, 49.6, $42.2,36.5,29.6,28.7,25.2$; ESIMS $m / z 607(M+1)$; HRMS calcd for: $\left(\mathrm{C}_{23} \mathrm{H}_{43} \mathrm{~N}_{8} \mathrm{O}_{11}+1\right), m / z$ (607.3046); found, $m / z$ (607.3358).

$\mathrm{N}$-(Phe-Asp-Gly-Arg)-2-amino-2-deoxy-a-D-glucosamine: The solution of $10 \mathrm{~b}(30 \mathrm{mg}, 0.026 \mathrm{mmol})$ in TFA $(2.1 \mathrm{~mL})$ and $\mathrm{CF}_{3} \mathrm{SO}_{3} \mathrm{H}$ $(0.7 \mathrm{~mL})$ was stirred at $0^{\circ} \mathrm{C}$ until all the starting material was consumed as indicated by TLC $(3 \mathrm{hr})$. The reaction mixture was added $\mathrm{Et}_{2} \mathrm{O}$ $(80 \mathrm{~mL})$, the precipitate was collected by filtration that was added $\mathrm{NH}_{3} \cdot \mathrm{H}_{2} \mathrm{O}$ to $\mathrm{pH}=9$. Subsequently, the residue was added $\mathrm{HAc}$ to $\mathrm{pH}=4$ and purified by column chromatography (Sephadex G-10) to provide Compound 11b (7.3 mg, $0.011 \mathrm{mmol}, 43 \%)$. IR ( $\mathrm{cm}^{-1}, \mathrm{KBr}$, neat): 3147, 1670; ${ }^{1} \mathrm{HNMR}\left(500 \mathrm{MHz}, \mathrm{DMSO}-d_{6}\right): \delta 4.69(1 \mathrm{H}, \mathrm{d}, \mathrm{J}=5.5 \mathrm{~Hz}$, $\mathrm{H}-1), 4.66(1 \mathrm{H}, \mathrm{m}, \mathrm{CH}), 4.52(1 \mathrm{H}, \mathrm{m}, \mathrm{CH}), 4.37(1 \mathrm{H}, \mathrm{m}, \mathrm{CH}), 4.14(2 \mathrm{H}$, $\mathrm{m}, \mathrm{CH}), 3.71(4 \mathrm{H}, \mathrm{m}, \mathrm{CH}), 3.36(2 \mathrm{H}, \mathrm{m}, \mathrm{CH}), 3.17\left(1 \mathrm{H}, \mathrm{m}, \mathrm{CH}_{2}\right), 3.08$ $\left(2 \mathrm{H}, \mathrm{m}, \mathrm{CH}_{2}\right), 2.77\left(1 \mathrm{H}, \mathrm{m}, \mathrm{CH}_{2}\right), 2.31\left(1 \mathrm{H}, \mathrm{m}, \mathrm{CH}_{2}\right), 2.29\left(1 \mathrm{H}, \mathrm{m}, \mathrm{CH}_{2}\right)$, $1.83\left(1 \mathrm{H}, \mathrm{m}, \mathrm{CH}_{2}\right), 1.55\left(3 \mathrm{H}, \mathrm{m}, \mathrm{CH}_{2}\right) ;{ }^{13} \mathrm{CNMR}\left(125 \mathrm{MHz}, \mathrm{CDCl}_{3}\right) \delta$ 176.1, 174.1, 173.9, 171.4, 160.7, 122.8, 122.4, 121.1, 119.8, 118.8, 116.4, $114.0,90.1,84.3,81.1,74.6,63.6,63.0,54.3,54.2,45.1,42.5,35.3,32.3$, 30.7, 24.8, 22.9; ESIMS $m / z$ 653(M-1); HRMS calcd for: $\left(\mathrm{C}_{27} \mathrm{H}_{43} \mathrm{~N}_{8} \mathrm{O}_{11}+1\right.$ ), $m / z$ (655.3046); found, $m / z$ (655.3363).

$\mathrm{N}$-(Ser-Asp-Gly-Arg)-2-amino-2-deoxy-a-D-glucosamine: The solution of $10 \mathrm{c}(30 \mathrm{mg}, 0.025 \mathrm{mmol})$ in TFA $(2.1 \mathrm{~mL})$ and $\mathrm{CF}_{3} \mathrm{SO}_{3} \mathrm{H}(0.7$ $\mathrm{mL}$ ) was stirred at $0^{\circ} \mathrm{C}$ until all the starting material was consumed as indicated by TLC $(3 \mathrm{hr})$. The reaction mixture was added $\mathrm{Et}_{2} \mathrm{O}(80 \mathrm{~mL})$, the precipitate was collected by filtration that was added $\mathrm{NH}_{3} \cdot \mathrm{H}_{2} \mathrm{O}$ to $\mathrm{pH}=9$. Subsequently, The residue was added $\mathrm{HAc}$ to $\mathrm{pH}=4$ and purified by column chromatography (Sephadex G-10) to provide Compound $11 \mathrm{c}(6.5 \mathrm{mg}, 0.011 \mathrm{mmol}, 44 \%)$. IR ( $\mathrm{cm}^{-1}, \mathrm{KBr}$, neat): 3194, 1701; ${ }^{1} \mathrm{HNMR}\left(300 \mathrm{MHz}, \mathrm{DMSO}-d_{6}\right): \delta 7.31(1 \mathrm{H}, \mathrm{brs}, \mathrm{N}-\mathrm{H}), 4.57(1 \mathrm{H}, \mathrm{m}$,
$\mathrm{CH}), 4.43(1 \mathrm{H}, \mathrm{m}, \mathrm{CH}), 4.31(3 \mathrm{H}, \mathrm{m}, \mathrm{H}-1, \mathrm{H}-2, \mathrm{H}-3), 4.08(3 \mathrm{H}, \mathrm{m}, \mathrm{CH}$ $\mathrm{H}-4, \mathrm{H}-5), 3.66\left(6 \mathrm{H}, \mathrm{m}, \mathrm{H}-6 \mathrm{a}, \mathrm{H}-6 \mathrm{~b}, \mathrm{CH}_{2}\right), 3.11\left(2 \mathrm{H}, \mathrm{m}, \mathrm{CH}_{2}\right), 2.60(2 \mathrm{H}$, $\left.\mathrm{m}, \mathrm{CH}_{2}\right), 1.79\left(2 \mathrm{H}, \mathrm{m}, \mathrm{CH}_{2}\right), 1.58\left(2 \mathrm{H}, \mathrm{m}, \mathrm{CH}_{2}\right) ;{ }^{13} \mathrm{CNMR}(125 \mathrm{MHz}$, $\left.\mathrm{CDCl}_{3}\right) \delta 176.1,174.1,173.9,171.4,160.7,122.8,122.4,121.1,119.8$, $118.8,116.4,114.0,90.1,84.3,81.1,74.6,63.6,63.0,54.3,54.2,45.1$, 42.5, 35.3, 32.3, 30.7, 24.8, 22.9; ESIMS $m / z$ 595(M+1); HRMS calcd for: $\left(\mathrm{C}_{21} \mathrm{H}_{39} \mathrm{~N}_{8} \mathrm{O}_{12}+1\right), m / z$ (595.2682); found, $m / z$ (595.2987).

\section{Results and Discussion}

\section{Chemistry}

As shown in Scheme 1, Boc- $\operatorname{Arg}\left(\mathrm{NO}_{2}\right)-\mathrm{OH}$ and glycine benzylester $\left(\mathrm{NH}_{2}\right.$-Gly-OBzl) were reacted using the standard peptide coupling method (dicyclohexylcarbodiimide (DDC), N-hydroxybenzotriazole (HOBt) and $\mathrm{N}$-methylmolpholine (NMM)) in solution followed by purification by silica gel column chromatography to afford dipeptide Boc- $\operatorname{Arg}\left(\mathrm{NO}_{2}\right)$-Gly-OBzl. Subsequently, removal of the benzyl group with $\mathrm{NaOH}$ resulted in Boc- $\mathrm{Arg}\left(\mathrm{NO}_{2}\right)$-Gly-OH (Compound 1) which was used to synthesize various tetrapeptides. The coupling reaction of L-Boc-Asp(OMe)-OH with different lipophilic amino acids benzylester, Val, Phe and Ser, yielded dipeptide Boc-Asp(OMe)-AAOBzl. Subsequently, removal of the Boc group with hydrogen chloride in ethyl acetate led to $\mathrm{N}$-terminal free intermediates (Compound 2ac) which was reacted with Compound 1 using the standard peptide coupling method (DDC, HOBt, and $N$-methylmolpholine) to give tetrapeptides (Comopunds 3a-c). Removal of the benzyl group from Compound $3 \mathrm{a}-\mathrm{c}$ was carried out by palladium catalyzed hydrogenolysis to give the corresponding $\mathrm{C}$-terminal free compound, (Compound 4ac), in almost quantitative yield.

The preparation of 2-amino-1,3,4,6-tetra-O-benzyl-2-deoxyD-glucopyranoside (Compound 8) [38] using glucosamine (GlcN) salt (chloride) as starting materials is presented in Scheme 2. Initially, acylation of $(\mathrm{GlcN})$ salt with (Boc) ${ }_{2} \mathrm{O}$ resulted in $\mathrm{N}$-Bocglucosamine (Compound 6). N-Boc-glucosamine was benzylated with $\mathrm{NaH}$ and $\mathrm{BnBr}$ to give 1,3,4,6-tetra-O-benzyl-2- (tertbutyloxycarbonylamino)-2-deoxy-D-glucopyranoside (Compound 7). Subsequently, removal of the Boc group with trifluoroacetic acid in dichloromethane led to 2-amino-1,3,4,6-tetra-O-benzyl-2deoxy-D-glucopyranoside (Compound 8) as mixture of anomers which was used for the facile preparation of mimetic peptides. The synthetic routes for the preparation of mimetic peptides from Compound 8 were presented in Schemes 3. Furthermore, Compound 8 and protected tetrapeptides Boc- $\operatorname{Arg}\left(\mathrm{NO}_{2}\right)$-GlyAsp $(\mathrm{OMe})-\mathrm{AA}-\mathrm{OH}$ (RGDAA, AA = amino acid residues) with various amino acid residues moiety as building blocks were explored to synthesize various glycosylated mimetic peptides by standard peptide coupling methods (DDC, HOBt and $N$-methylmolpholine) in solution phase. The products obtained were purified by silica gel column chromatography to afford $9 a-c$ in $69 \%, 79 \%$ and $61 \%$ yield,

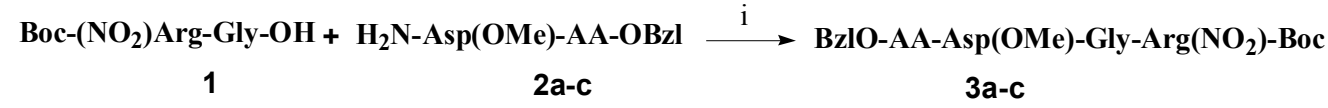

ii

HO-AA-Asp(OMe)-Gly-Arg( $\left.\mathrm{NO}_{2}\right)$-Boc

4a-c

$\begin{array}{r}\mathbf{A A}=\text { Val } \\ \text { Phe } \\ \text { Ser } \\ \hline\end{array}$

Scheme 1: Synthesis of tetrapeptides. Reagents and conditions: (i)NMM, DDC, HOBt; (ii) H2 ,Pd/C. 


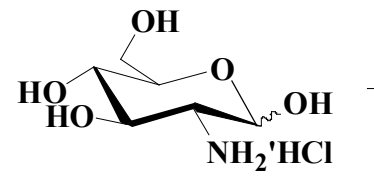

5

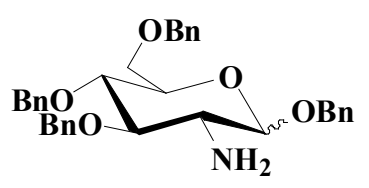

8

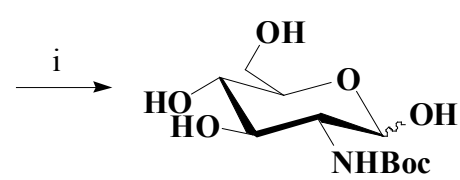

6
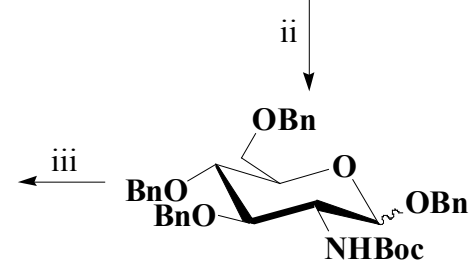

7

Scheme 2: Synthesis of 2-amino-1,3,4,6-tetra-O-benzyl-2-deoxy-D-glucosamine. Reagents and conditions: (i) $\mathrm{Boc}_{2} \mathrm{O}$ and triethylamine; (ii) BnBr, NaH, DMF (iii) $\mathrm{CF}_{3} \mathrm{COOH}$.

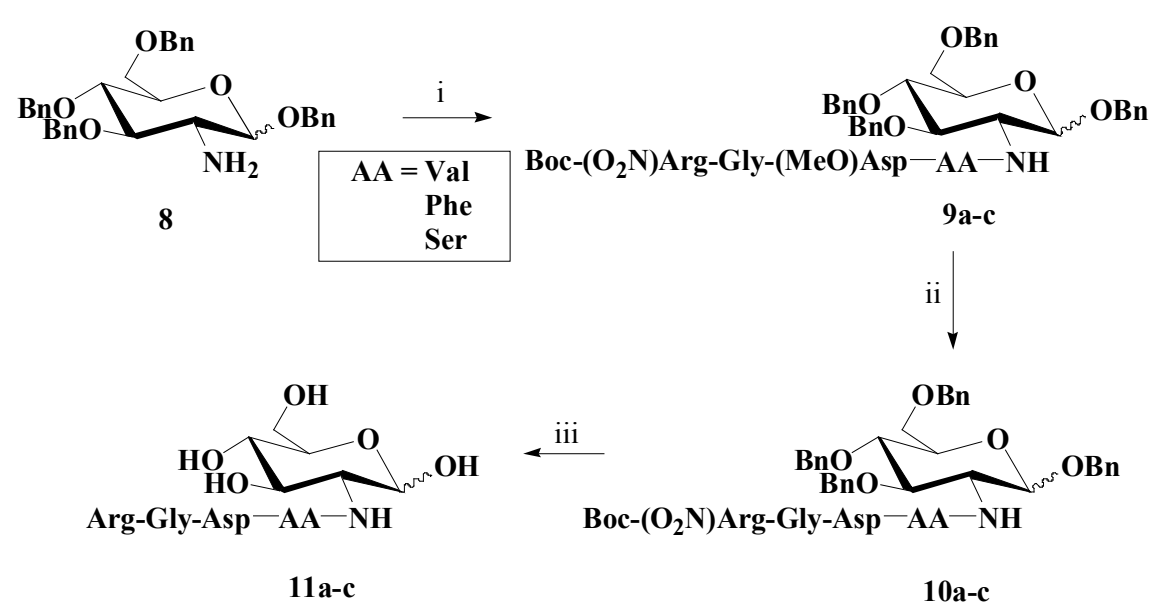

Scheme 3: Synthesis of mimetic Peptides containing glucosamine.Reagents and conditions: (i)NMM, DDC, $\mathrm{HOBt}, \mathrm{HO}-\mathrm{AA}-\mathrm{Asp}(\mathrm{OMe})-\mathrm{Gly}-\mathrm{Arg}\left(\mathrm{NO}{ }_{2}\right)-\mathrm{Boc}$; (ii) $\mathrm{NaOH}$; (iii) $\mathrm{CF}_{3} \mathrm{COOH}, \mathrm{CF}_{3} \mathrm{SO}_{3} \mathrm{H}$.

respectively. The assignment of the configuration was performed by ${ }^{1}$ HNMR spectroscopy. The ${ }^{1}$ HNMR spectra of 9a showed signals for the anomeric protons as doublets with coupling constants of 4.0 $\mathrm{Hz}$ and $8.5 \mathrm{~Hz}$ as a mixture of anomers $(\alpha: \beta=1.8: 1) .{ }^{1} \mathrm{HNMR}$ spectra of $9 b-c$ showed signals for the anomeric protons as doublets with coupling constants of $3.0 \mathrm{~Hz}$ and $3.5 \mathrm{~Hz}$ as single anomers, which can be attributed to the anomeric effect. Subsequently, removal of the Me group with $\mathrm{NaOH}$ led to a set of carboxylic acid 10a-c in $94 \%, 70 \%$ and $75 \%$ yield, respectively. ${ }^{1} \mathrm{HNMR}$ spectra indicated that $10 \mathrm{a}$ was as a mixture of anomers which were separated by silica gel column chromatography to afford $\alpha$-anomer and $\beta$-anomer in $74 \%$ and $20 \%$ yield, respectively $(\alpha: \beta=3.7: 1)$ and $10 \mathrm{~b}-\mathrm{c}$ were $\alpha$-anomers. Problems were encountered at the removal of Boc group of $10 \mathrm{a}-\mathrm{c}$ where using hydrogen chloride in ethyl acetate is failed. Unfortunately, the attempt to remove nitro and benzyl groups by palladium catalyzed hydrogenolysis resulted in only an intractable mixture of several products. Finally, Compound 11a-c presented were obtained following the removal of the Boc group, benzyl groups and nitro group with trifluoromethanesulfonic acid in trifluoroacetic acid as a mixture of antiomers in $38 \%, 43 \%$ and $44 \%$ yield respectively. The structures of glycopeptides are shown in Table 1.

\section{Evaluation of anti-inflammatory activity}

xylene-induced mouse ear edema are the typical animal models used to evaluate potential anti-inflammatory agents. They have a good reputation for screening anti-inflammatory agents. Compounds 5, $9 a-c, 10 a-c, 11 a-c$ were screened for their anti-inflammatory activity by using a xylene-induced ear edema model assay [39]. The animal protocol was approved by the Committee on Animal Care and Usage (Capital Medical University). Male ICR mice (body weight, 18-20 g) were used. The animals were maintained on a 12/12 h light/dark cycle at constant temperature and humidity, and provided with free access to food and water in the home cage. They were allowed to acclimate to their new surroundings for 1 day before experiment. Mice were divided into eight groups of twelve. Mice administered orally with $0.5 \%$ $\mathrm{CMC}$ were used as the negative control group, and mice administered orally with Aspirin (at a dosage of $100 \mathrm{mg} / \mathrm{kg}$ ) in CMC were used as positive control. The compounds to be tested were prepared as fine homogenized suspensions in $0.5 \% \mathrm{CMC}$ at the concentration of $1 \mathrm{mM}$ and were administered orally to the animals at a dosage of $10 \mu \mathrm{mol} /$ $\mathrm{kg}$ body weight $30 \mathrm{~min}$ before xylene was applied to both the anterior and posterior surfaces of the right ear. The left ear was considered as control. Two hours after xylene treatment, all mice were decapitated 


\begin{tabular}{|c|c|c|c|}
\hline No & Chemical structure & No & Chemical structure \\
\hline $9 a$ & Boc- $\left(\mathrm{O}_{2} \mathrm{~N}\right) \operatorname{Arg}-\mathrm{Gly}-(\mathrm{MeO}) \mathrm{Asp}-\mathrm{Val}-\mathrm{NH}$ & $9 b$ & Boc- $\left(\mathrm{O}_{2} \mathrm{~N}\right)$ Arg-Gly-(MeO)Asp-Phe-NH \\
\hline $9 c$ & Boc- $\left(\mathrm{O}_{2} \mathrm{~N}\right)$ Arg-Gly-(MeO)Asp- $(\mathrm{BnO}) \mathrm{Ser}-\mathrm{NHOBn}_{\mathrm{Bn}}$ & $10 \mathrm{a}(\alpha)$ & Boc- $\left(\mathrm{O}_{2} \mathrm{~N}\right) \mathrm{Arg}-\mathrm{Gly}-\mathrm{Asp}-\mathrm{Val}-\mathrm{NH}_{\mathrm{OBn}}$ \\
\hline $10 \mathrm{a}(\beta)$ & Boc- $\left(\mathrm{O}_{2} \mathrm{~N}\right) \mathrm{Arg}-\mathrm{Gly}-\mathrm{Asp}-\mathrm{Val}-\mathrm{NH}$ & $10 \mathrm{~b}$ & Boc- $\left(\mathrm{O}_{2} \mathrm{~N}\right)$ Arg-Gly-Asp-Phe- $\mathrm{NH}_{\mathrm{OBn}}$ \\
\hline $10 \mathrm{c}$ & $\begin{array}{r}\mathrm{BnO} \\
\mathrm{Bn} \\
\mathrm{Boc}-\left(\mathrm{O}_{2} \mathrm{~N}\right) \text { Arg-Gly-A }\end{array}$ & $11 a$ & $\mathrm{H}_{2} \mathrm{~N}$-Arg-Gly-Asp- $\mathrm{Val}-\mathrm{NH}$ \\
\hline $11 \mathrm{~b}$ & $\mathrm{H}_{2} \mathrm{~N}-$ Arg-Gly-Asp-Phe-NH & $11 \mathrm{c}$ & $\mathrm{H}_{2} \mathrm{~N}$-Arg-Gly-Asp-Ser- \\
\hline
\end{tabular}

Table 1: Structures of glucosamine mimetic peptides derivatives.

\begin{tabular}{|c|c|c|}
\hline Compound $^{a}$ & Edema weight $(X \pm S D$ mg $)$ & Inhibition ratio (\%) \\
\hline CMC & $7.6 \pm 2.8$ & $31.8 \pm 23.9$ \\
\hline Aspirin & $0.9 \pm 0.6$ & $89.5 \pm 7.0^{c}$ \\
\hline 5 & $3.5 \pm 2.2$ & $64.3 \pm 23.0^{b}$ \\
\hline $9 a$ & $1.2 \pm 1.1$ & $87.5 \pm 11.8^{c}$ \\
\hline $9 b$ & $2.6 \pm 1.6$ & $78.5 \pm 13.1^{b}$ \\
\hline $9 c$ & $1.7 \pm 1.1$ & $86.8 \pm 9.0^{c}$ \\
\hline $10 a-\alpha$ & $1.9 \pm 1.5$ & $84.5 \pm 12.1^{b}$ \\
\hline $10 a-\beta$ & $2.0 \pm 1.4$ & $81.1 \pm 15.7^{b}$ \\
\hline 10b & $1.6 \pm 1.3$ & $86.7 \pm 10.5^{c}$ \\
\hline $10 c$ & $1.2 \pm 0.9$ & $90.7 \pm 7.1^{c}$ \\
\hline $11 a$ & $2.4 \pm 1.4$ & $82.6 \pm 11.0^{b}$ \\
\hline 11b & $1.9 \pm 1.3$ & $85.7 \pm 9.9^{c}$ \\
\hline 11c & $1.7 \pm 0.8$ & $86.9 \pm 6.3^{c}$ \\
\hline
\end{tabular}

${ }^{a}$ Aspirin=Positive control, $\mathrm{CMC}=$ Vehicle, Dose of mimetic peptides derivatives $=10 \mu \mathrm{mol} / \mathrm{kg}$,

dose of aspirin = $100 \mathrm{mg} / \mathrm{kg} ; \mathrm{n}=12$.

${ }^{b}$ Compared to $\mathrm{CMC} p<0.01$.

${ }^{\circ}$ Compared to CMC $p<0.01$, Compared to $5 p<0.05$.

Table 2: Anti-inflammatory activities of glucosamine mimetic peptides derivatives determined using an xylene-induced ear edema model.

by diethyl ether anesthesia and both ears were removed and weighed. The increase in weight caused by xylene was measured through subtracting the weight of the untreated left ear section from that of the treated right ear section. The inhibition ratio (\%) in describing the antiinflammatory effects was determined:

\section{Inhibition ratio $\%=(1-\mathrm{A} / \mathrm{B}) \times 100 \%$}

A: The increase in weight caused by xylene

B: The weight of the untreated left ear section

The efficacies of these derivatives anti-inflammatory activity were compared with the positive control and the negative control, respectively. The percentage of inhibition is used as an indication of anti-inflammatory activity. The anti-inflammatory activity of these derivatives is summarized in Table 2 . The results revealed that all the derivatives tested significantly reduced ear edema and exhibited a significant inhibitory activity against xylene-induced inflammation in mice in comparison with the negative control group. The tested derivatives showed inhibition ratio ranging from $78.5 \%$ to $90.7 \%$ ( $\mathrm{P}<0.01)$ with Compound 10c being most effective. The ear edema inhibition ratio for Compound $10 \mathrm{c}$ at $10 \mu \mathrm{mol} / \mathrm{kg}$ was calculated to be $90.7 \%$, while that was $89.5 \%$ for aspirin at the dosage of $100 \mathrm{mg} / \mathrm{kg}$. Inhibition ratio of compound $10 \mathrm{c}$ was clearly better than that produced by aspirin. To understand the contribution of the tetrapeptides (RGDAA) containing RGD in glucosamine mimetic peptides to the anti-inflammatory activities, glucosamine 5 was used as reference compounds. Comparison of inhibition ratio values of tested derivatives and glucosamine $(64.3 \%)$ showed that the presence of tetrapeptides can increased the activity, suggesting that the tetrapeptides structure had a definite contribution to the activity. Further investigation is required on the biological activity of these compounds in order to rationalize these observations. This study provided useful information for the further design of novel potent agents. 


\section{Conclusions}

In summary, a novel class of glucosamine mimetic peptides containing bioactive RGD peptides derivatives were designed, synthesized and evaluated for their anti-inflammatory effect. It was found that Compounds $10 \mathrm{c}$ exhibited the most potent antiinflammatory activity. The tetrapeptides structure containing RGD had a definite contribution to the activity.

\section{Acknowledgements}

This work was supported by the National Natural Scientific Foundation of China (20642004), the Educational Council Foundation of Beijing (KM200710025012), the National Basic Research Program of China (2011CB504100), the National Natural Scientific Foundation of China (81030062) and Key National Science \& Technology Specific Projects (2011ZX09102-003-01). Academic Human Resources Development in Institutions of Higher Learning Under the Jurisdiction of Beijing Municipality (PHR201007114), Beijing Pharmacological Society (2013BI01)

\section{References}

1. Yao N, Fung G, Malekan H, Ye L, Kurth MJ, et al. (2010) Facile synthesis of glycosylated Fmoc amino acid building blocks assisted by microwave irradiation. Carbohydr Res 345: 2277-2281.

2. Inaba Y, Kawakami T, Aimoto S, Ikegami T, Takeuchi T, et al. (2009) Preparation and conformational analysis of C-glycosyl beta(2)- and beta/beta(2)-peptides. Carbohydr Res 344: 613-626.

3. Dwek RA (1996) Glycobiology: Toward Understanding the Function of Sugars Chem Rev 96: 683-720.

4. Varki A (1993) Biological roles of oligosaccharides: all of the theories are correct. Glycobiology 3: 97-130.

5. Wong C (1999) Mimics of complex carbohydrates recognized by receptors. Chem Res 32: 376 -385.

6. Herzner H, Reipen T, Schultz M, Kunz H (2000) Synthesis of glycopeptides containing carbohydrate and Peptide recognition motifs. Chem Rev 100: 44954538.

7. Lis H, Sharon N (1993) Protein glycosylation. Structural and functional aspects. Eur J Biochem 218: 1-27.

8. Collins BE, Paulson JC (2004) Cell surface biology mediated by low affinity multivalent protein-glycan interactions. Curr Opin Chem Biol 8: 617-625.

9. Berger EG, Buddecke E, Kamerling JP, Kobata A, Paulson JC, et al. (1982) Structure, biosynthesis and functions of glycoprotein glycans. Experientia 38: 1129-1162.

10. Blithe DL (1993) Biological functions of oligosaccharides on glycoproteins. Trends Glycosci. Glycotech. 5: 81-98.

11. Takeuchi M, Takasaki S, Shimada M, Kobata A (1990) Role of sugar chains in the in vitro biological activity of human erythropoietin produced in recombinant Chinese hamster ovary cells. J Biol Chem 265: 12127-12130.

12. Takeuchi M, Inoue N, Stlckiand TW, Kubota M, Wada M, et al. (1989) Relationship between sugar chain structure and biological activity of recombinant human erythropoietin produced in Chinese hamster ovary cells. USA Proc Natl Acad Sci 86: 7819-7822.

13. Jennings HJ (1990) Capsular polysaccharides as vaccine candidates. Curr Top Microbiol Immunol 150: 97-127.

14. Jennings HJ (1988) Chemically modified capsular polysaccharides as vaccines. Adv Exp Med Biol 228: 495-550.

15. Lee CJ (1987) Bacterial capsular polysaccharides--biochemistry, immunity and vaccine. Mol Immunol 24: 1005-1019.

16. Weintraub A (2003) Immunology of bacterial polysaccharide antigens Carbohydr Res 338: 2539-2547.

17. Wang LX, Ni J, Singh S, Li H (2004) Binding of high-mannose-type oligosaccharides and synthetic oligomannose clusters to human antibody 2G12: implications for HIV-1 vaccine design. Chem Biol 11: 127-134.

18. Johnson MA, Pinto BM (2002) Molecular Mimicry of Carbohydrates by Peptides. Aust J Chem 55: 13-25.

19. Routenberg LK, Seeberger PH (2004) Automated Solid-Phase Synthesis of Protected Tumor-Associated Antigen and Blood Group Determinant Oligosaccharides. Angew Chem 116: 612-615.

20. Plante OJ, Palmacci ER, Seeberger PH (2003) Development of an automated oligosaccharide synthesizer. Adv Carbohydr Chem Biochem 58: 35-54.

21. Kelly GS (1998) The role of glucosamine sulfate and chondroitin sulfates in the treatment of degenerative joint disease. Altern Med Rev 3: 27-39.

22. Xing R, Liu S, Guo Z, Yu H, Li C, et al. (2006) The antioxidant activity of glucosamine hydrochloride in vitro. Bioorg Med Chem 14: 1706-1709.

23. Arnaout MA, Goodman SL, Xiong JP (2002) Coming to grips with integrin binding to ligands. Curr Opin Cell Biol 14: 641-651.

24. Plow EF, Haas TA, Zhang L, Loftus J, Smith JW (2000) Ligand binding to integrins. J Biol Chem 275: 21785-21788.

25. Trabocchi A, Menchi G, Cini N, Bianchini F, Raspanti S, et al. (2010) Clickchemistry-derived triazole ligands of arginine-glycine-aspartate (RGD) integrins with a broad capacity to inhibit adhesion of melanoma cells and both in vitro and in vivo angiogenesis. J Med Chem 53: 7119-7128.

26. Varner JA, Cheresh DA (1996) Integrins and cancer. Curr Opin Cell Biol 8 : 724-730.

27. Yin RT, Zheng $H, X i T, X u$ HM (2010) Effect of RGD-4C Position is More Important than Disulfide Bonds on Antiangiogenic Activity of RGD-4C Modified Endostatin Derived Synthetic Polypeptide. Bioconjugate Chem 21: 1142-1147.

28. Getlik M, Grutter C, Simard JR, Kluter S, Rabiller M, et al. (2009) Hybrid compound design to overcome the gatekeeper T338M mutation in cSrc. J Med Chem 52: 3915-3926.

29. Morphy R, Kay C, Rankovic Z (2004) From magic bullets to designed multiple ligands. Drug Discov Today 9: 641-651.

30. Natarajan A, Guo Y, Harbinski F, Fan YH, Chen H, et al. (2004) Nove arylsulfoanilide-oxindole hybrid as an anticancer agent that inhibits translation initiation. J Med Chem 47: 4979-4982.

31. Romagnoli R, Baraldi PG, Tabrizi MA, Bermejo J, Estevez F, et al. (2005) Design, synthesis, and biological evaluation of hybrid molecules containing alpha-methylene-gamma-butyrolactones and alpha-bromoacryloyl moieties. J Med Chem 48: 7906-7910.

32. Baraldi PG, Romagnoli R, Guadix AE, Pineda de las Infantas MJ, Gallo MA et al. (2002) Design, synthesis, and biological activity of hybrid compounds between uramustine and DNA minor groove binder distamycin A. J Med Chem 45: 3630-3638.

33. Watanabe A, Kai T, Nagase $H$ (2006) Novel synthesis of the ortho ester derivative of 4,5-epoxymorphinan. Org Lett 8: 523-526.

34. Dal Pozzo A, Ni MH, Esposito E, Dallavalle S, Musso L, et al. (2010) Nove tumor-targeted RGD peptide-camptothecin conjugates: synthesis and biological evaluation. Bioorg Med Chem 18: 64-72.

35. Seo J, Michaelian N, Owens SC, Dashner ST, Wong AJ, et al. (2009) Chemoselective and microwave-assisted synthesis of glycopeptoids. Org Lett 11: $5210-5213$.

36. Zhang J, Zhao M, Peng S (2011) Synthesis of mimetic peptides containing glucosamine. Carbohydr Res 346: 1997-2003.

37. Zeng L, Zhang J (2012) Design, synthesis, and evaluation of a novel class of 2,3-disubstituted-tetrahydro- $\left.\right|^{2}$-carboline derivatives. Bioorg Med Chem Lett 22: 3718-3722.

38. Agarwal J, Peddinti RK (2011) Glucosamine-based primary amines as organocatalysts for the asymmetric aldol reaction. J Org Chem 76: 3502-3505.

39. Li X, Zhao M, Tang YR, Wang C, Zhang Z, et al. (2008) N-[2-(5,5-dimethyl,3-dioxane-2-yl)ethyllamino acids: their synthesis, anti-inflammatory evaluation and QSAR analysis. Eur J Med Chem 43: 8-18. 